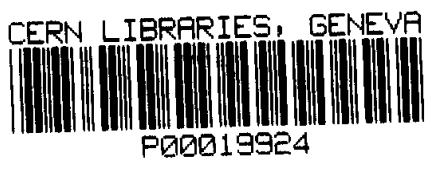

CERN-PPE/93-210

1 December 1993 849402

\title{
Performance of an Electromagnetic Liquid Krypton Calorimeter
}

\section{Fanti}

Dipartimento di Fisica dell'Universitá e Sezione dell'INFN di Cagliari, 09100 Cagliari, Italy

G. D. Barr, P. Buchholz, D. Cundy, N. Doble, L. Gatignon, A. Gonidec, B. Hallgren,

G. Kesseler, A. Lacourt, G. Laverrière, G. Linser, A. Norton, D. Schinzel, W. Seidl,

T. Stemmler ${ }^{1)}, \mathrm{H}$. Taureg, G. Viehhauser and H. Wahl CERN, 1211 Geneva 23, Switzerland

J. Duclos, A. Gianoli, M. Martini ${ }^{2}$, L. Piemontese, and M. Savrié

Dipartimento di Fisica dell'Universitá e Sezione dell'INFN di Ferrara, 44100 Ferrara, Italy

A. Kalinin, J. Kekelidze and Y. Kozhevnikov

Joint Institute for Nuclear Research, Dubna, Russia

$$
\text { D. Coward }{ }^{3)} \text {, F. Leber }
$$

Institut für Physik, Universität Mainz, 55099 Mainz, Germany ${ }^{4)}$

P. Cenci, P. Lariccia, P.Lubrano and M. Pepe

Dipartimento di Fisica dell'Universitá e Sezione dell'INFN di Perugia, 06100 Perugia, Italy

P. Calafiura, C. Cerri, R. Fantechi ${ }^{2)}$, B. Gorini, F. Laico, I. Mannelli, V. Marzulli and D. Schiuma

Dipartimento di Fisica dell'Universitá e Scuola Normale Superiore e Sezione dell'INFN di Pisa, 56010 Pisa, Italy

P. Debu, E. Mazzucato, A. Migliori, B. Peyaud and R. Turlay CE-Saclay-DAPNIA, 91191 Gif-sur-Yvette, France

\section{A. Kreutz}

Fachbereich Physik, Universität Siegen, 57068 Siegen, Germany ${ }^{5)}$

C. Biino, A. Ceccucci and S. Palestini ${ }^{2)}$

Dipartimento di Fisica Sperimentale dell'Universitá e Sezione dell'INFN di Torino, 10125 Torino, Italy

E. Griesmayer ${ }^{2)}$, M. Markytan, G. Neuhofer, M. Pernicka, T. Taurok and C.-E. Wulz Österreichische Akademie der Wissenschaften, Institut für Hochenergiephysik, 1050 Wien, Austria

\section{Cagliari-Cambridge-CERN-Dubna-Edinburgh-Ferrara-Mainz- Perugia-Pisa-Saclay-Siegen-Torino-Vienna Collaboration}




\begin{abstract}
A prototype electromagnetic calorimeter containing 180 litres of liquid krypton has been tested in electron and muon beams at the CERN SPS. The main features of this detector are its active shower medium, a granularity of $2 \mathrm{~cm}$ with tower read out, and the use of the initial current readout technique with an effective shaping time of about 60 ns. An energy resolution for electrons of $4.1 \% / \sqrt{E[\mathrm{GeV}]}$ with a constant term of $0.4 \%$ and a noise contribution of $50 \mathrm{MeV}$ has been achieved. The calorimeter has a spatial resolution of $4.5 \mathrm{~mm} / \sqrt{E[\mathrm{GeV}]}$ with a constant term of $0.3 \mathrm{~mm}$ and a time resolution of about $0.5 \mathrm{~ns}$ for energies larger than $10 \mathrm{GeV}$.
\end{abstract}

1) Present address : E-PLUS Mobilfunk GmbH, Nürnberg, Germany.

2) Present address : CERN, 1211 Geneva 23, Switzerland.

3) On leave from SLAC, Stanford, CA 94305, USA.

4) Funded by German Federal Minister for Research and Technology (BMFT) under contract 05 4MZ18.

5) Funded by German Federal Minister for Research and Technology (BMFT) under contract 055 SI74. 
Introduction

This collaboration is preparing a new precision measurement of direct $\mathrm{CP}$ violation in $K^{0} \rightarrow 2 \pi$ decays at CERN [1]. The aim of the experiment is to determine the parameter $\epsilon^{\prime} / \epsilon$ with an accuracy of $2 \times 10^{-4}$. The main features of the experiment are the concurrent detection of all four decay modes $\left(K_{L} \rightarrow \pi^{0} \pi^{0}, K_{L} \rightarrow \pi^{+} \pi^{-}, K_{S} \rightarrow \pi^{0} \pi^{0}, K_{S} \rightarrow \pi^{+} \pi^{-}\right)$ in the same detector, nearly collinear $K_{L}$ and $K_{S}$ beams (which are distinguished by tagging the proton which produces the $K_{S}$ particles), a magnetic spectrometer to reduce the background for the $K_{L} \rightarrow \pi^{+} \pi^{-}$decays to less than one part in a thousand, a fast liquid krypton calorimeter for the detection of photons from $\pi^{0}$ decay with excellent energy and space resolution to reduce background for the $K \rightarrow \pi^{0} \pi^{0}$ decays, a $40 \mathrm{MHz}$ flash ADC readout and a fully pipelined trigger using the energy, centre of gravity and second moment measured in the calorimeter.

It is planned to run with ten times the beam intensity and event rates compared to the previous CP violation experiment at CERN [2]. Correspondingly, systematic uncertainties will have to be reduced by at least a factor of three.

\subsection{Performance Requirements of the Electromagnetic Calorimeter}

The proposed electromagnetic calorimeter has an active volume of liquid krypton with an octagonal fiducial area $2.4 \mathrm{~m}$ across (diameter of an inscribed circle). The depth is 27 radiation lengths corresponding to $1.25 \mathrm{~m}$ of liquid krypton. The singles rate is expected to be about $1 \mathrm{MHz}$. The photon energy range is 2 to $100 \mathrm{GeV}$ with an average of about $25 \mathrm{GeV}$. The energy resolution is required to be significantly better than $7.5 \% / \sqrt{E[\mathrm{GeV}]}$ as achieved previously [2]. The pulse height response has to be calibrated and stable to better than one per mille. The space resolution should be better than $1 \mathrm{~mm}$ and it should be possible to resolve two photons separated by more than about $4 \mathrm{~cm}$. The time resolution should be better than $1 \mathrm{~ns}$ for photon energies above $10 \mathrm{GeV}$.

To achieve these design goals, a liquid krypton calorimeter of high granularity with a $2 \mathrm{~cm} \times 2 \mathrm{~cm}$ readout tower structure is being built. Altogether there will be about 13500 towers. This segmentation ensures the high rate capability, good space resolution and photon separation. The use of liquid krypton in a quasi-homogeneous structure (the only other materials are thin readout electrodes) minimizes sampling fluctuations as needed to achieve the excellent energy resolution required. The total volume of liquid krypton will be about $8 \mathrm{~m}^{3}$ (19 tons). Low detector capacitance of about $80 \mathrm{pF}$ per cell and the use of cold preamplifiers with short connections result in a high speed readout. The initial current readout technique $[3,4]$ is used with a pulse shaping of 40 ns peaking time and total width of about $160 \mathrm{~ns}$ (the total drift time across the gap has been measured to be $3.0 \mu$ s at $3000 \mathrm{~V}[5])$.

\subsection{Properties of Liquid Krypton}

Table 1 shows some relevant properties of liquid krypton. For the electron drift velocity and lifetime in liquid krypton see also section 2.4. Krypton contains a radioactive isotope, ${ }^{85} \mathrm{Kr}$, which is a $\beta^{-}$emitter with a maximum energy of $670 \mathrm{KeV}$ and a half-life of 10.74 years. The radioactivity of the krypton used was measured with an ionization 
chamber to be about $500 \mathrm{~Bq} / \mathrm{cm}^{3}[6]$.

\begin{tabular}{|l|c|}
\hline$Z$ & 36 \\
$A$ & 84 \\
$\rho\left(\mathrm{g} / \mathrm{cm}^{3}\right)$, density at $120^{\circ} \mathrm{K}$ & 2.41 \\
$X_{0}(\mathrm{~cm})$, radiation length & 4.7 \\
$R_{M}(\mathrm{~cm})$, Molière radius & 4.7 \\
$W(\mathrm{eV} /$ pair $)$, energy to create one ion-electron pair & 20.5 \\
$\lambda_{I}(\mathrm{~cm})$, nuclear interaction length & 60 \\
$T_{b}\left({ }^{\circ} \mathrm{K}\right)$, boiling point at 1 bar & 119.8 \\
$T_{m}\left({ }^{\circ} \mathrm{K}\right)$, melting point & 116.0 \\
$v_{d}^{e}(\mathrm{~mm} / \mu \mathrm{s})$, electron drift velocity at $1(5) \mathrm{kV} / \mathrm{cm}$ & $2.7(3.7)$ \\
$E_{c}(\mathrm{MeV})$, critical energy & 21.51 \\
\hline
\end{tabular}

Table 1: Physical properties of liquid krypton.

\section{The Prototype Calorimeter}

A cylindrical prototype calorimeter with a volume of $39 \mathrm{~cm}$ diameter and $140 \mathrm{~cm}$ depth containing about 1801 liquid krypton and with the granularity as planned for the final calorimeter has been built and tested in an electron beam. Preliminary results have been reported previously $[7,8]$.

\subsection{Readout Structure}

Two readout structures have been tested, both based on the principle of a tower structure of cells formed by thin electrodes stretched in the beam direction. They differed in the electrode thickness and the way the preamplifier and the high voltage were connected.

The first structure consisted of flat vertical Kapton foils of $125 \mu \mathrm{m}$ thickness stretched with a tension of about $600 \mathrm{~N}$ per foil. On the foils the tower cells were defined by $19 \mathrm{~mm}$ wide copper strips separated by $1 \mathrm{~mm}$. The thickness of the copper cladding was $17 \mu \mathrm{m}$ plus about $10 \mu \mathrm{m}$ of glue on each side of the foil. The distance between foils was $1 \mathrm{~cm}$. Each $2 \times 2 \mathrm{~cm}^{2}$ tower cell consisted of two half cells defined by three foils, one foil on either side of a central foil. The length of each tower was $120 \mathrm{~cm}$. There were 184 calorimeter cells in total. Figure 1 shows the foil structure.

The preamplifier was directly attached to the foil structure in the liquid krypton, where electrons drifted from both sides to the centre foil. The connection scheme for the first readout structure (shown in figure 2) used high voltage of alternating polarity in order to use the foil as the high voltage decoupling capacitor and to avoid discrete decoupling capacitors in the cold. Since in this approach the high voltage was applied across the Kapton foil, samples had been tested with high voltage both in air at room temperature and in liquid argon. The $125 \mu \mathrm{m}$ foils held $10 \mathrm{kV}$ in liquid argon for more than a month without breakdown. Before assembly, all the foils of the prototype had been tested at $10 \mathrm{kV}$ in a nitrogen atmosphere with a relative humidity below $40 \%$ and showed no 


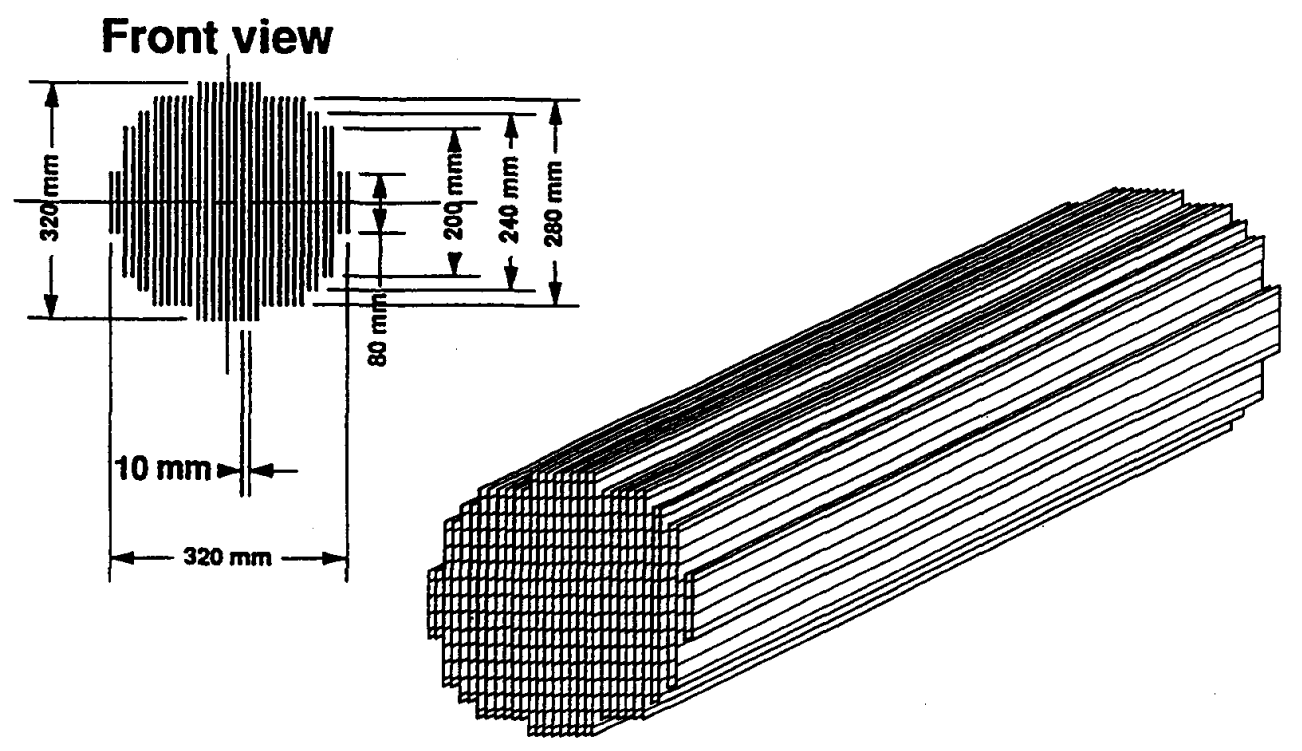

Figure 1: Readout foil structure of the prototype calorimeter.

problems. After assembly, problems due to a deterioration of the foils in regions where they were glued to other materials led to high voltage breakdowns. Therefore a second readout structure with a different connection scheme was constructed. It used commercial high voltage decoupling capacitors mounted directly on the readout structure in the liquid krypton (shown in figures 3 and 4 ).

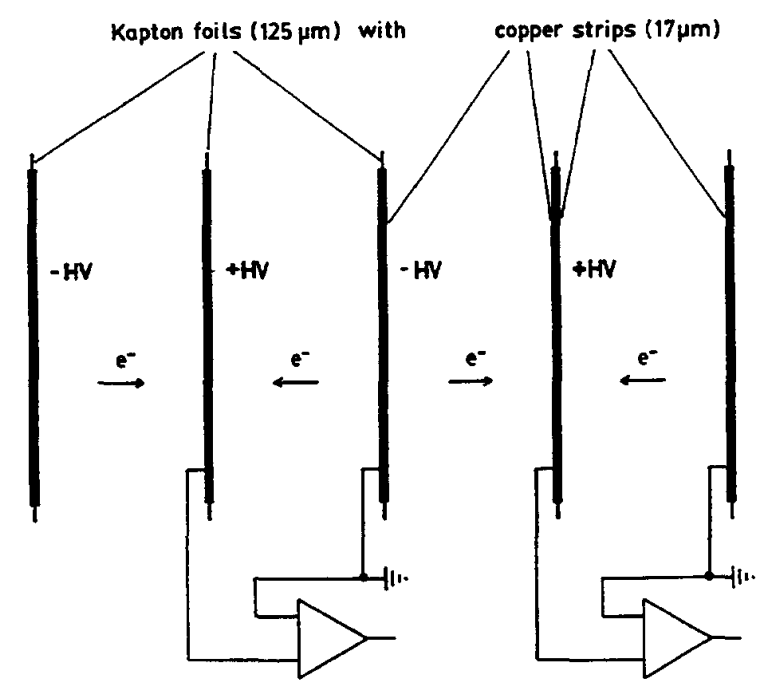

Figure 2: Readout cell layout of the first prototype calorimeter.

The second prototype structure had the same segmentation as the previous structure but with $125 \mathrm{~cm}$ long tower cells. The towers were again made of etched copper cladded Kapton foils but this time Kapton foils of $75 \mu \mathrm{m}$ thickness were used. An individual tower is shown in figure 4 together with its electrical connections. The foils were held 
at the front and the back of the calorimeter by frames, as shown in figure 3. A saw-tooth shaped front frame was built in order to minimize the amount of krypton between the front frames of adjacent foils, hence achieving better uniformity of the material thickness in front of the active krypton. The distance between the foils was $1 \mathrm{~cm}$ and each foil was stretched with $15 \mathrm{~N} / \mathrm{cm}$. Slotted spacers of $1 \mathrm{~mm}$ thick Stesalit material were inserted like combs every $30 \mathrm{~cm}$ to define a constant gap of $1 \mathrm{~cm}$. The slots were cut with a water-jet to a width of $200 \mu \mathrm{m}$. In order to investigate a possible deterioration in resolution, additional half-combs were placed in between the fixed combs at $15 \mathrm{~cm}, 45 \mathrm{~cm}$, etc., covering only the left half of the calorimeter structure. The first part of the test run was done using this configuration and since no left/right difference in resolution could be found, but better uniformity was observed where more combs were present, the calorimeter was equipped with full combs every $15 \mathrm{~cm}$ for the remaining beam time.

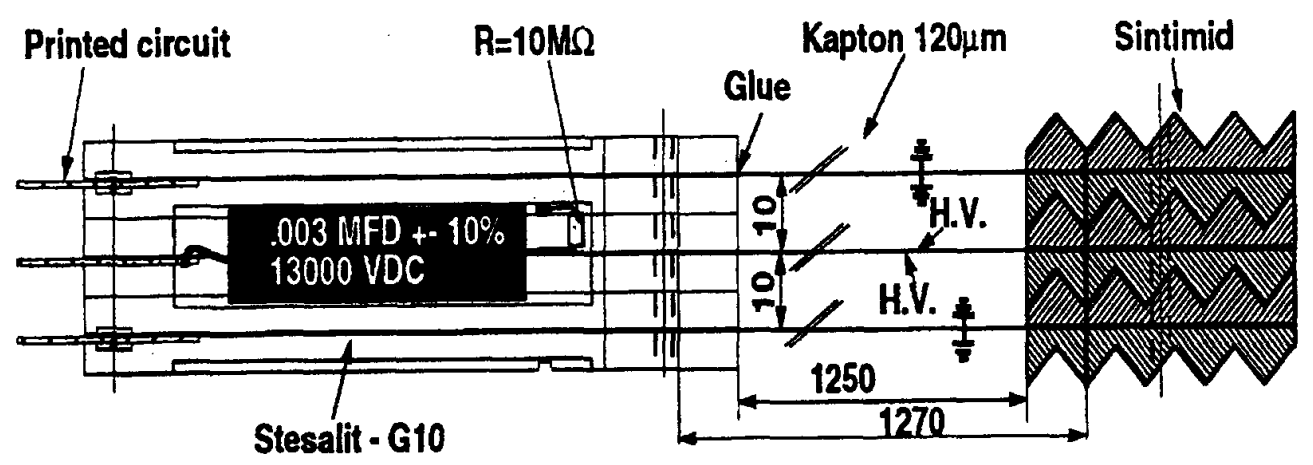

Figure 3: Front and back frames of the second readout structure.

Every even-numbered foil was kept at ground potential and every odd-numbered foil was connected to high voltage. High voltage was distributed using one bus per foil. Each individual strip of a Kapton foil was connected to its bus with a $10 \mathrm{M} \Omega$ resistor. All buses were joined together and connected to the outside of the cryostat via two cable feedthroughs. The preamplifier inputs were decoupled from the HV strips using $3 \mathrm{nF} \mathrm{HV}$ capacitors ${ }^{1)}$. The capacitors used in the calorimeter were tested beforehand in liquid nitrogen up to $8 \mathrm{kV}$ and their values were measured. The average value found at liquid nitrogen temperature was $2.95 \mathrm{nF}$ with a spread of $1.5 \%$. HV buses and capacitors were housed in special frames to use the same electronics as for the first prototype structure.

Most of the data were taken with a high voltage of $+3 \mathrm{kV}$ applied to the $\mathrm{HV}$ strips. For the measurement of the high voltage dependence of the calorimeter response the voltage was increased to a maximum of $+5 \mathrm{kV}$ (see section 4.1.5).

1) ASC, American Shizuki Corporation, Ogallala, Nebraska, USA. 


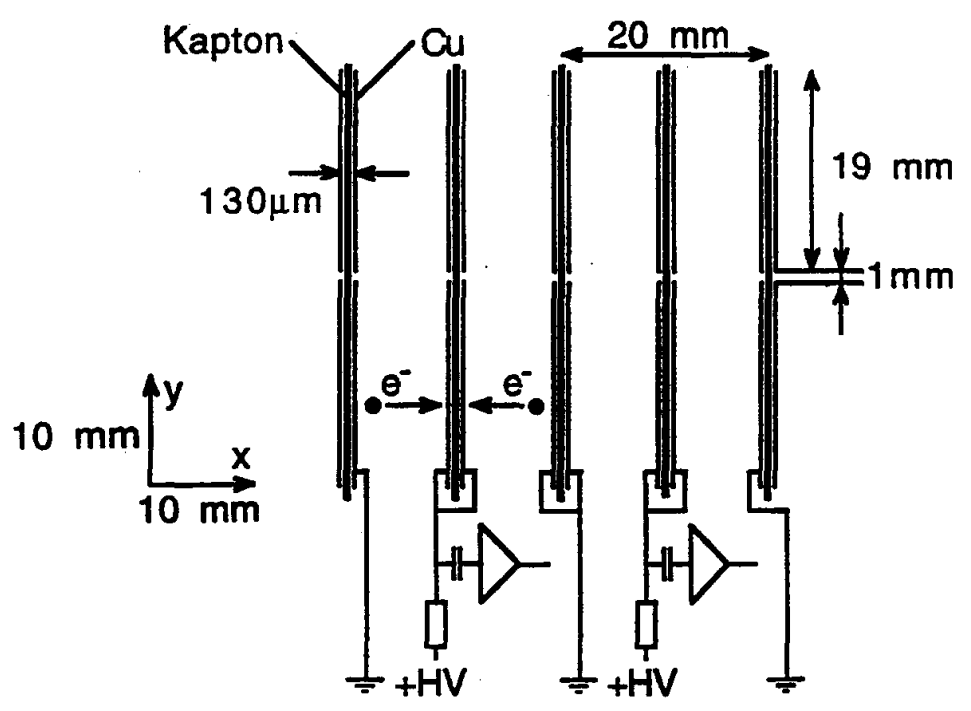

Figure 4: Readout cell layout of the second prototype calorimeter.

\subsection{Cryogenic System}

A simplified flow diagram of the cryogenic system is shown in figure 5 . The vacuum insulated cryostat with the calorimeter test module contained approximately 180 litres of liquid krypton, which was kept at slight overpressure with respect to atmosphere, typically 1.2 bar (all pressures given here are absolute); the boiling temperature of the liquid was then $122^{\circ} \mathrm{K}$. The static thermal input to the cryostat of some $70 \mathrm{~W}$ evaporated around 1 litre of liquid per hour. The evaporated krypton was warmed to room temperature, sent through a gas purifier (which could be by-passed by a valve) and then into the vacuum insulated krypton cooler for re-liquefaction.

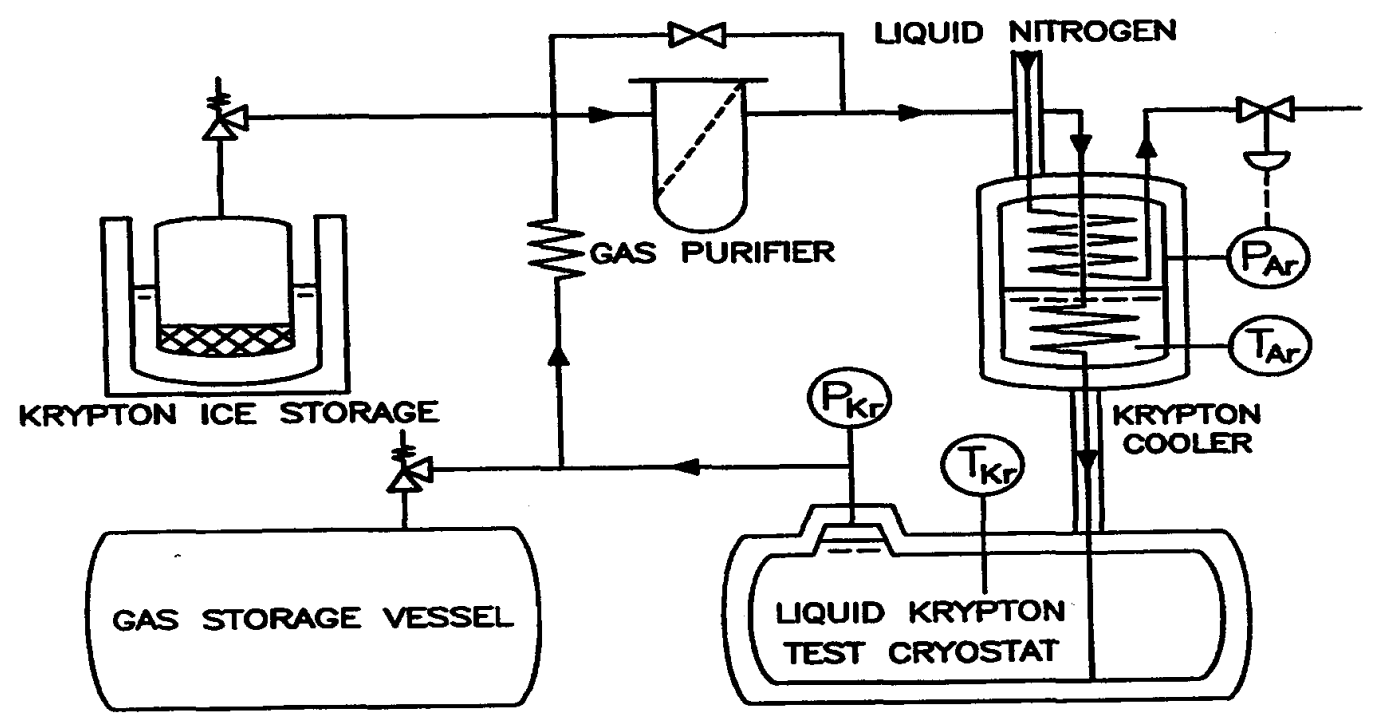

Figure 5: Simplified flow diagram of the cryogenic system. 
The cooler was maintained at a fixed temperature slightly above the triple point of krypton $\left(116^{\circ} \mathrm{K}\right.$ ) using a bath of liquid argon pressurised to about 10 bar (the corresponding temperature is $116.6^{\circ} \mathrm{K}$ ). The argon in turn was cooled with liquid nitrogen flowing through a heat exchanger in the gas space of the vessel and controlled by the argon pressure.

The characteristics of the cooling principle are sketched in figure 6 , which shows the vapour pressure curves of both argon and krypton. At a given pressure $P_{A r}$ in the argon container, the temperature of the liquid will be $T_{\boldsymbol{A} r}$. The liquefaction process requires a temperature difference $\Delta T$ between the krypton and the argon bath, consequently the liquefied krypton leaves the cooler at the temperature $T_{K r}$ and the pressure in the cryostat will adjust to $P_{K r}$. Due to the positive slope of the liquid krypton vapour pressure curve, a rise in $P_{K r}$ will cause $\Delta T$ to increase and therefore increase the krypton liquefaction rate. Since a liquefaction rate increase will cause $P_{K r}$ to fall, the equilibrium of the system is stable. Thus the desired pressure in the krypton cryostat can be tuned by setting $P_{A r}$ correspondingly.

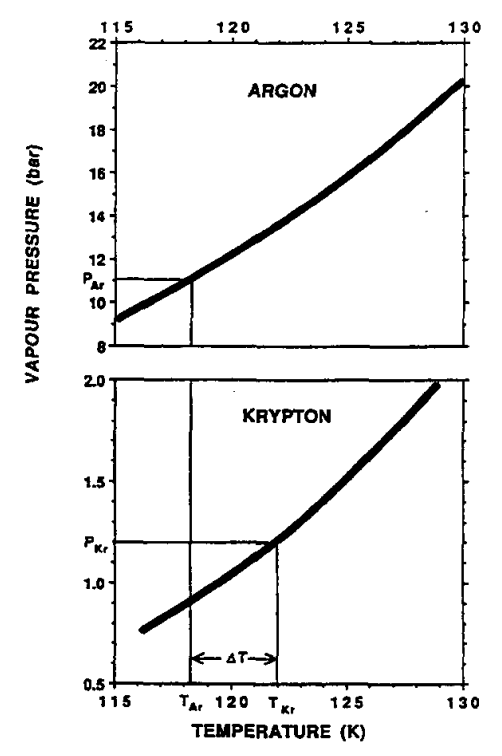

Figure 6: Characteristics of the cooling principle.

Two separate krypton storage techniques were provided to minimize the possibility of inadvertently losing the krypton. The normal storage consisted of a metal barrel permanently immersed in liquid nitrogen in which the krypton could be stored as ice. If the ice storage had failed, the krypton would have been retained after conversion to warm gas by a $40 \mathrm{~m}^{3}$ gas storage pressure vessel which was kept evacuated during normal operation.

\subsection{Gas Purification System}

The purification system is shown in figure 7. It consisted of commercially available Oxisorb and Hydrosorb filters ${ }^{2)}$. The gas was delivered in individual bottles. It was passed

2) Messer Griesheim $A G$ 
through the filters and liquefied into two 220 litre stainless steel barrels. All transfer lines and vessels were carefully cleaned and baked out before use. The complete system was evacuated and leak tested down to $10^{-9} \mathrm{mbar} \mathrm{l} / \mathrm{s}$ to avoid contamination with air during the purification. To remove dust particles coming from the filters, the transfer line in front of the receiving barrels contained a self-made dust filter with a pore size of $0.5 \mu \mathrm{m}$. Each barrel was mounted inside a Dewar which was filled with liquid nitrogen so that the krypton froze after liquefaction. Liquid nitrogen was automatically topped up during krypton storage. The flow rate of krypton gas through the filters and the pressures before and after the filters of the gas purification system were monitored. In this way, conditions could be kept constant and the amount of liquefied krypton was known.

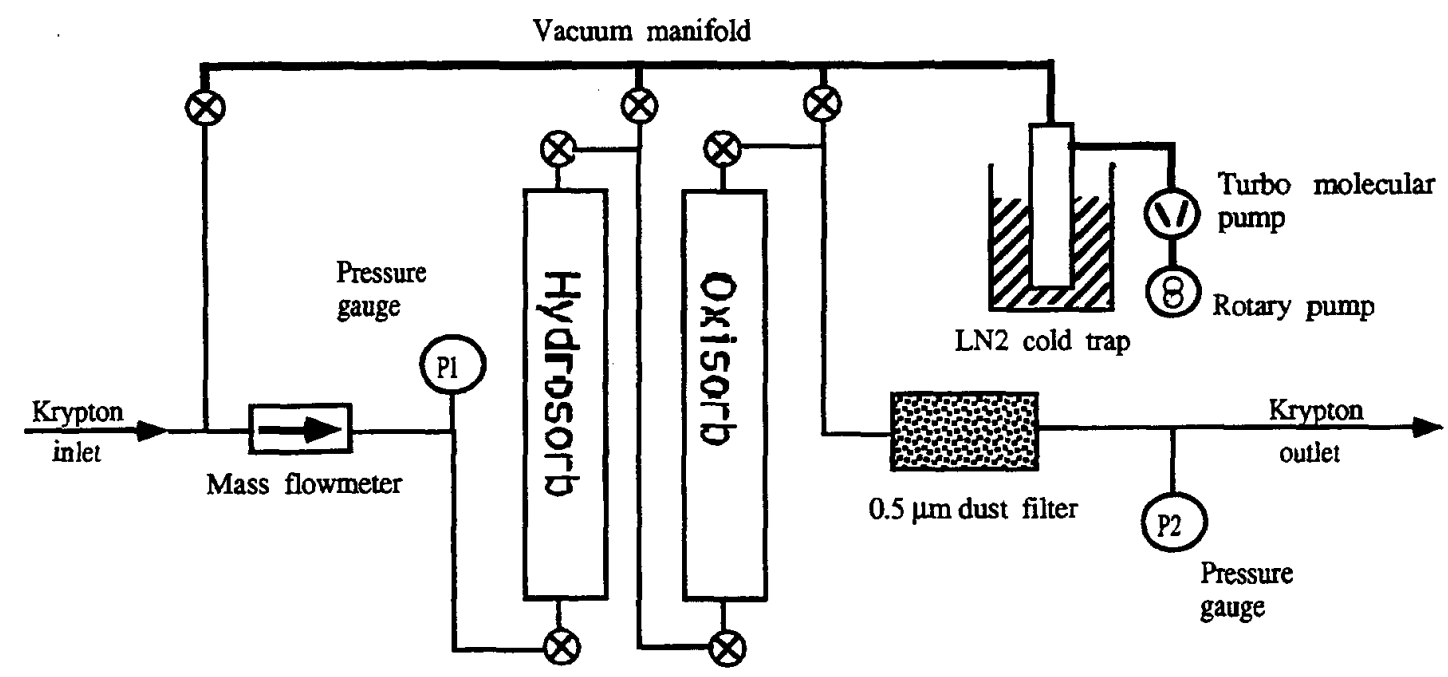

Figure 7: Purification system of the prototype calorimeter.

During the purification, samples could be transferred into a special counter so that the drift velocity and free electron lifetime could be measured. Free electrons were created either by the passage of minimum ionizing particles (cosmic rays) or by photoelectric emission from a metallic electrode using a Nd-Yag laser with quadrupler [9]. Both methods yielded typical lifetimes in the range of 40 to $100 \mu \mathrm{s}$ (figure 8 ).

The barrels containing the krypton ice blocks were equipped with heaters to facilitate liquefaction and subsequent evaporisation whenever needed. In order to fill the calorimeter for a test run, the liquid nitrogen of one barrel was removed from the corresponding Dewar and the heater was switched on. During the heating the vapour pressure was constantly monitored. At typically 1.8 bar the transfer to the krypton cooler of the calorimeter was started. Above 1.8 bar the heater of the barrel was automatically switched off and below-1.4 bar it came back on again. During normal operation the heating power was sufficient to compensate the heat of vaporisation and no regulation occurred.

The evaporated gas was passed through the purifier. The flow rate through the purifier and the pressure before and after the purifier (i.e. the pressure in the calorimeter cryostat were monitored). A second dust filter was mounted immediately in front of the 

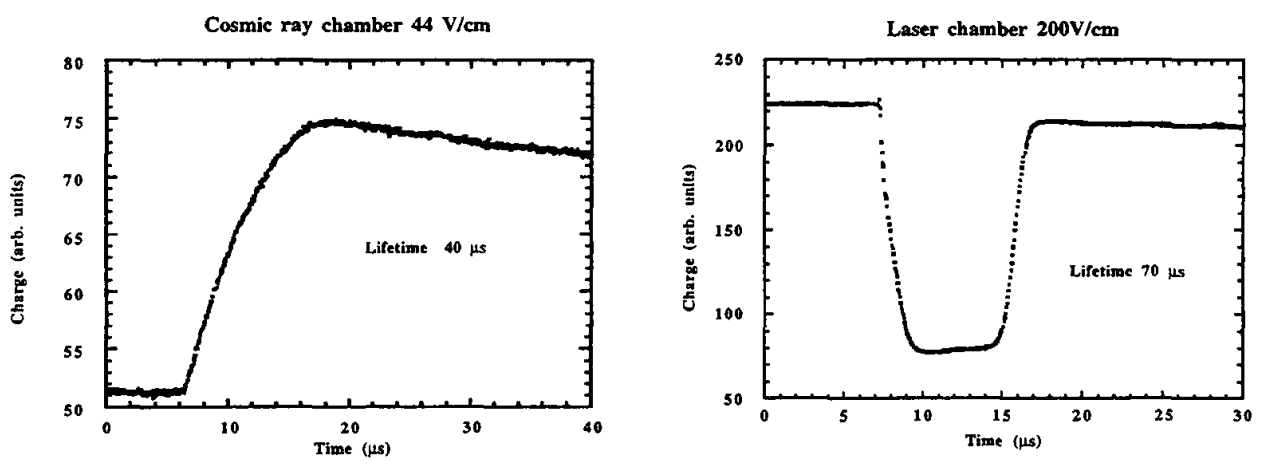

Figure 8: Typical electron lifetime.

krypton cooler. Typically 180 litres of liquid krypton were transferred through the purifier into the calorimeter in about 12 hours. This limitation in flow rate was due to the cooling power of the krypton cooler and not to the impedance of the filters.

The purification system was mounted so that boil-off gas could enter the krypton cooler either through the purifier (online purification mode) or directly, bypassing the purifier. Since the krypton remained pure enough during the run, no online purification was needed. During the filling of the calorimeter, the electron lifetime decreased by about a factor 10 but after filling remained stable throughout the run.

\subsection{Electron Lifetime and Drift Velocity}

Since both electron lifetime and drift velocity are of crucial importance in a fast, high resolution liquid krypton calorimeter, both were measured and monitored throughout the test run.

Uniform ionization across a tower of the calorimeter leads to a triangular current pulse whose length is given by the drift time $t_{d}$. Long electron lifetimes, typically $2 t_{d}$ to $3 t_{d}$ long, are necessary to avoid distortions of the output pulse shape of the shaping circuitry which was designed for very long electron lifetime. For a short electron lifetime, the expected current would be modified by the lifetime term $\exp (-t / \tau)$

$$
I_{(t)}=\frac{N e}{t_{d}}\left(1-t / t_{d}\right) \exp (-t / \tau)
$$

and the shaper response would deviate in height, time and (to a lesser extent) in width.

The drift velocity $v_{d}$ must also be known accurately because the drift time $t_{d}$ strongly affects the current $I_{(t)}$ generated in one tower. For the liquid krypton inside the calorimeter, both quantities, the lifetime $\tau$ and the drift time $t_{d}$, have to be determined from a single measurement (i.e. the integral of the current $I_{(t)}$ as a function of time). The output of the preamplifier was digitised in an transient recorder ${ }^{3)}$ and analysed during the run. After correcting for the impact point dependence of the pulse shape and using a drift time of $3 \mu \mathrm{s}$ [5], a lower limit of $3 \mu \mathrm{s}$ could be given for the lifetime [6].

3) LeCroy Research Systems, Model 6841. 
In order to get an independent result, a sample was taken from the full cryostat several hours after the filling and the electron lifetime in this sample was determined in the laboratory. The measurement yielded a lifetime larger than $6 \mu \mathrm{s}$. After two weeks of running, a second sample was taken from the cryostat and again yielded a lifetime larger than $6 \mu \mathrm{s}$.

\subsection{Electronic Readout System}

In order to achieve a fast readout, charge sensitive preamplifiers based on a design by V.Radeka et al. [10] were directly mounted on the readout structure and immersed in liquid krypton. They were mounted on an eight layer printed circuit board which also held the calibration system (see section 2.6). The readout capacitance was $80 \mathrm{pF}$ per calorimeter cell. There was an additional $30 \mathrm{pF}$ due to each of the vertically adjacent readout strips. The preamplifier signal was fed to a shaper circuit with two differentiation and four integration time constants [11]. The output pulse had a rise time of about $50 \mathrm{~ns}$ and a total length of about 200 ns. There was an undershoot lasting as long as the drift across the gap $(3 \mu \mathrm{s})$ with an amplitude of $5 \%$ of the peak signal. The shaper had three outputs. The first shaper output of each calorimeter cell was connected via a pulse splitter to peak sensing $\mathrm{ADCs}^{4)}$ as well as to charge integrating $\mathrm{ADCs}^{5}$ ). In addition, up to 80 cells were equipped with $40 \mathrm{MHz}$ linear 8 bit FADCs ${ }^{6}$ ) using the second shaper output. The third output was used for trigger tests. Figure 9 shows a schematic of one readout cell with the electronics.

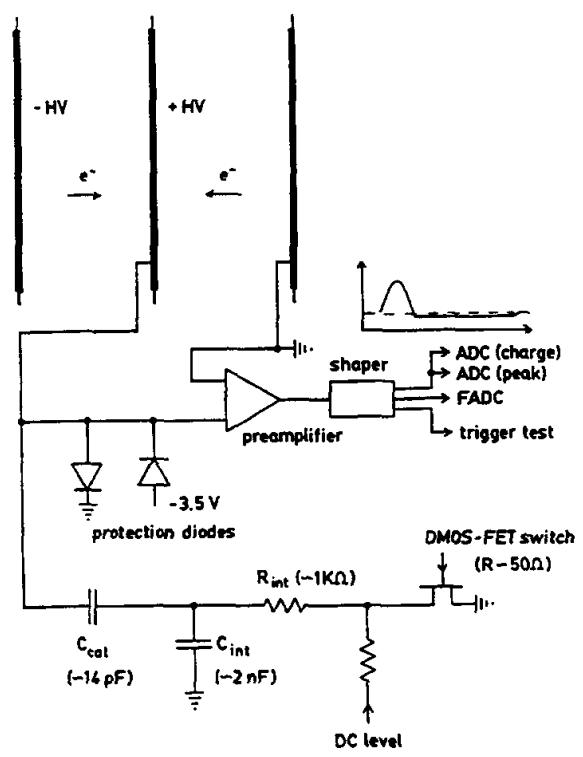

Figure 9: Readout cell with electronics of the prototype calorimeter.

4) LeCroy Research Systems, Model 2281 .

5) LeCroy Research Systems, Model 2282.

6) Dr. Bernd Struck, STR750 Flash-ADC System. 


\subsection{Calibration System}

The calibration system is shown in figure 9. Calibration pulses of initial current values determined by an input $D C$ voltage were produced with a DMOS-FET switch and an $\mathrm{RC}$ circuit integrated in the preamplifier motherboard. Calibration data were taken each beam burst interspersed with beam data.

All capacitances and resistances of the calibration system were measured at room temperature and liquid nitrogen temperature. At liquid krypton temperature they are known to better than one percent accuracy, including long term stability effects.

The linearity of the electronic readout chain was measured to be better than $0.2 \%$ using the calibration system.

\section{Test Beam Setup \\ 3.1 Beam Line}

The prototype calorimeter was installed in the $\mathrm{H} 4$ beam line at the CERN Super Proton Synchroton (SPS). It was exposed to electron, pion and muon beams. Data were taken with electron beam energies in the range 10 to $120 \mathrm{GeV}$. The momentum bite of the electron beam was $\sigma(E) / E=0.5 \% / \sqrt{E}$, the reproducibility of the beam energy after changing of the settings was estimated to be $\Delta E / E=3.2 \% / E$ and the absolute energy was known to $\Delta E / E \approx 25 \% / E \oplus 0.5 \%$. The pion contamination of the electron beam at normal intensity was estimated to be a few percent at low energies and smaller than one percent at energies above $50 \mathrm{GeV}$.

\subsection{Trigger}

The electron trigger was obtained from the signals of two scintillation counters, slightly smaller in diameter than the entrance window of the cryostat $(10 \mathrm{~cm})$, placed $1.25 \mathrm{~m}$ and $2 \mathrm{~m}$ upstream of the calorimeter. Particles entering the cryostat outside the window region were vetoed by a counter $1.9 \mathrm{~m}$ upstream with a central hole of $8.5 \mathrm{~cm}$ diameter. The muon trigger was formed by the coincidence of the signals of a counter $2 \mathrm{~m}$ upstream and another counter $4.5 \mathrm{~m}$ downstream of the calorimeter. To achieve a good time resolution, constant fraction discriminators were used in the electronics setup of the trigger. By comparing the two trigger counter signals the resolution on the time of the electron trigger signal was measured to 180 ps.

\subsection{Impact Point Reconstruction}

The impact point of the incoming particle on the calorimeter front face was reconstructed using two high precision drift chambers with 4 sense wire planes ( 2 horizontal and 2 vertical) each. The chambers were placed $0.9 \mathrm{~m}$ and $2.2 \mathrm{~m}$ upstream of the calorimeter. With $100 \mu \mathrm{m}$ resolution per chamber, the resolution on the electron impact point on the calorimeter front face was about $190 \mu \mathrm{m}$ at $50 \mathrm{GeV} / \mathrm{c}$. At lower energies multiple scattering in the scintillator trigger counters became important and at $10 \mathrm{GeV} / \mathrm{c}$ the impact point resolution was about $470 \mu \mathrm{m}$. 


\subsection{Electronic Noise}

The electronic noise was measured by summing the ADC pedestals independently for all even numbered calorimeter channels and all odd numbered channels. The noise was dominated by random fluctuations, with a significant contribution from coherent pedestal variations. These contributions were separated by comparing the RMS of the distribution of the sum and the RMS of the distribution of the difference of even and odd numbered channels.

This analysis has been performed for all three ADC systems used. With the peak sensing and charge integrating $\mathrm{ADC}$ systems the electronic noise was measured to be $46 \mathrm{MeV}$ for a shower box of $11 \times 11$ cells. In order to increase the dynamic range of the FADC system, up to three FADCs with different gain settings were connected to the same cell (see section 4.3. Each gain type contributed differently to the electronic noise. The total noise in a shower box using all 80 cells equipped with FADCs was measured to be $60 \mathrm{MeV}$. The electronic noise was monitored during the run and was stable within $\pm 5 \%$.

\section{$4 \quad$ Results}

\subsection{Energy Resolution}

\subsubsection{Uniformity of the Calorimeter Response}

Non-uniformities in the response of the calorimeter affect its performance if they cannot be corrected by calibration. In the case of a liquid krypton calorimeter with a tower readout structure the electromagnetic shower profile is rather narrow and very steep near its axis. The average shower profile as measured with $120 \mathrm{GeV}$ electrons is shown in figure 10. Up to $50 \%$ of the visible shower energy is deposited in the central calorimeter cell.

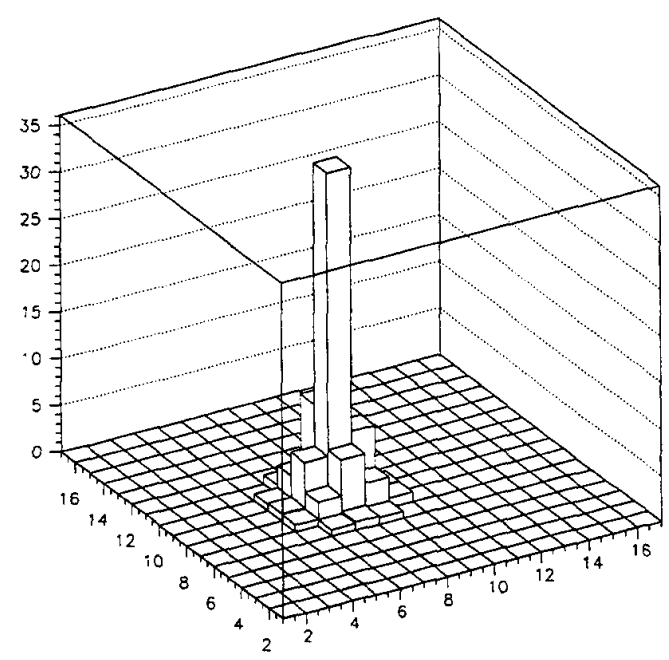

Figure 10: Typical shower profile as measured with $120 \mathrm{GeV}$ electrons.

There are two main sources for a non-uniformity of the calorimeter response with tower readout. The first is the mechanical tolerance of the cell width and the second is given by the presence of the charge collecting electrodes. These electrodes are made of 
passive material and only part of the charge produced close to the electrodes is seen due to the effective pulse shaping time of about $60 \mathrm{~ns}$. Both effects would average out in case of uniform ionization across the calorimeter. But, this is not the case for a shower profile as shown in figure 10. Both a left-right asymmetry in the two half-cells forming one calorimeter cell and a decrease of the charge close to the readout electrodes were observed in the data. In the following, both effects are discussed in more detail.

For cells with an uniform electric drift field the initial current $I^{0}$ is given by

$$
I^{0}=\frac{q \times v_{d}}{w}
$$

where $q$ is the total ionization charge deposited in the cell, $v_{d}$ the drift velocity and $w$ the width of a cell. In the case of the showers perpendicular to the direction of drift and a cell size of the order of half a Molière radius, the density of the ionization is changing strongly over the width of a cell. Then the precision with which the cell width $w$ is known can become a limiting factor in the resolution of the calorimeter. Since each cell consists of two half-cells, deviations from the nominal width tend to be opposite for adjacent half-cells and left-right asymmetries in the two half-cells can be observed.

Figure 11 shows the response of the calorimeter in one cell as a function of $x$, the coordinate of the electron impact point perpendicular to the readout electrodes, determined using the drift chamber information. This cell was chosen since it showed the largest observed left-right asymmetry.

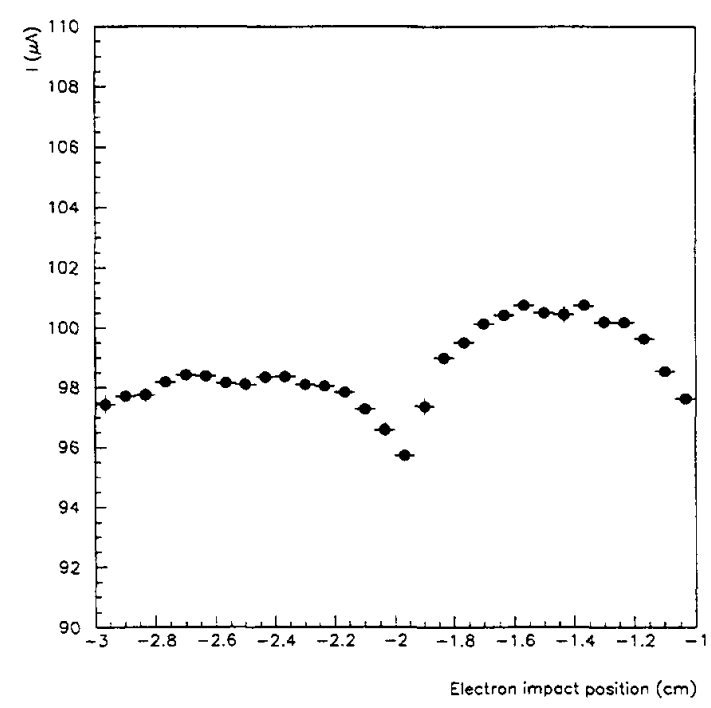

Figure 11: Cell 76 response as a function of the electron impact position.

During the last period of the test run, extra spacers were inserted to improve the constancy of the foil spacing. The improvement of the response uniformity can be seen in figure 12 . 


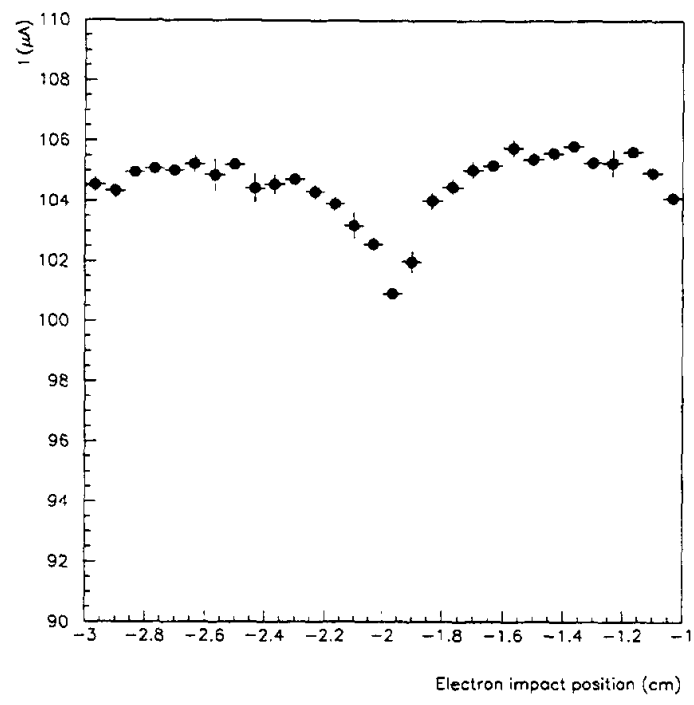

Figure 12: Cell 76 response as a function of the electron impact position after insertion of extra spacers.

Monte Carlo simulation [12] confirmed that in order to maintain the energy independent term in the resolution to less than $0.5 \%$, the mechanical tolerances defining the width of the cells must be kept to better than $1 \%$ (i.e. $\pm 100 \mu \mathrm{m}$ ) for the geometry of this prototype.

The second effect is related to the fraction of ionization deposited in the passive material of the electrodes and to the effective shaping time $t_{m}$ during which the initial current is measured. Since $t_{m}$ is of the order of $60 \mathrm{~ns}$, the ionization deposited in a region distant from the anode by less than $\Delta x=t_{m} \times v_{d}$ does not fully contribute to the initial current measurement. As a consequence, the current loss is small if the shower core is far away from the anode, but a larger amount of charge is lost if the particle impinges close to the anode. Independently of integration time, a larger fraction of ionization ends in passive material when the shower is closer to either anode or cathode. As a result, the response of the calorimeter as a function of the impact position shows depletions at the positions of the electrodes. These depletions are less pronounced at the cathodes.

To minimize non-uniformities, a small angle zig-zag of the readout electrodes is planned for the final calorimeter. In order to study the benefits of a small angle between the calorimeter cell structure and the beam axis, data were taken at calorimeter orientations of 0,13 and $26 \mathrm{mrad}$ with respect to the beam axis. The depletion present at the centre of the cell is reduced by this rotation. Typical values for the depletion are reported in table 2 . The tilt smeared out and hence decreased the non-uniformities.

The remaining non-uniformities have been measured and subsequently corrected offline for the determination of the final energy resolution. 


\begin{tabular}{|l|c|c|}
\hline Tilt angle (mrad) & Cell 76 & Cell 77 \\
\hline 0 & $2.5 \%$ & $3.0 \%$ \\
13 & $1.8 \%$ & $2.0 \%$ \\
26 & $1.2 \%$ & $1.8 \%$ \\
\hline
\end{tabular}

Table 2: Calorimeter response non-uniformity near the readout electrode position as a function of the tilt angle.

\subsubsection{Energy Resolution}

The energy resolution is obtained from a Gaussian fit to the energy distribution measured by summing pulse heights in a shower box of $11 \times 11$ calorimeter cells around the shower maximum. A typical energy spectrum is shown in figure 13. Calibration and non-uniformity corrections have been applied to obtain the final energy resolution of the calorimeter. The resolution after calibration but before any non-uniformity corrections is shown in figure 14 as a function of energy.

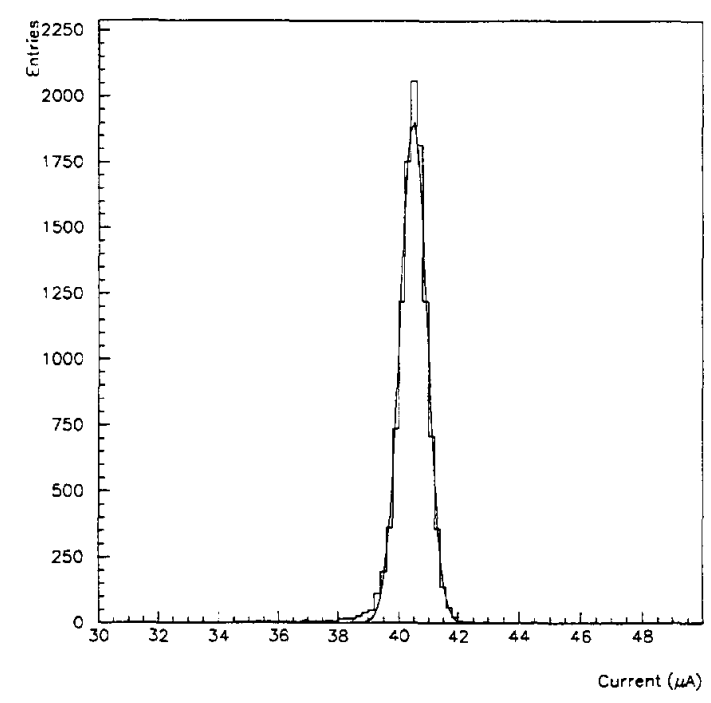

Figure 13: A typical energy spectrum for electrons of $20 \mathrm{GeV}$.

The final energy resolution was obtained by correcting non-uniformities of the response in fine steps of $0.5 \mathrm{~mm}$ along the $\mathrm{x}$ coordinate. Each cell was divided into 40 regions along $x$ and the average response was measured for each bin. Different data sets were used for the determination of the correction factors and for the measurement of the energy resolution. The correction factors were calculated at a fixed energy and applied to all data since no significant energy dependence was observed. The correction factor which was applied was chosen as a function of the centre of gravity of the shower in the calorimeter. The data used were taken with the prototype calorimeter tilted by $26 \mathrm{mrad}$ with respect to the beam axis.

The energy resolution can be parametrized as 


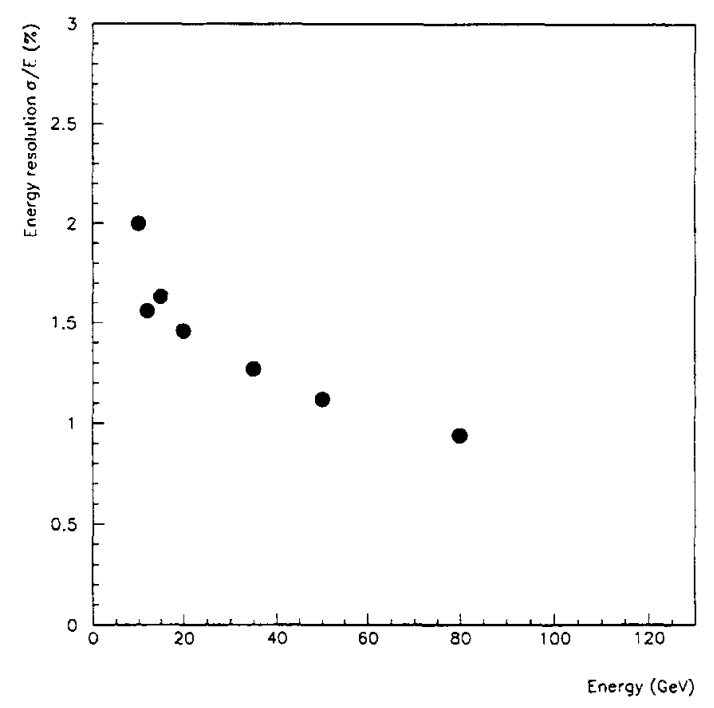

Figure 14: Energy resolution of the prototype calorimeter as measured for different electron energies before non-uniformity corrections.

$$
\frac{\sigma}{E}=\sqrt{\frac{A^{2}}{E}+\frac{B^{2}}{E^{2}}+C^{2}} .
$$

In order to evaluate the statistical shower fluctuation term $A$, we limited the analysis to electrons populating only one of the central cells of the prototype. In this configuration the effect of cell-to-cell intercalibration inaccuracy was negligible and $A$ was determined by a fit imposing the constant term as $C=0$ and the noise contribution $B$ as $50 \mathrm{MeV}$. The fit is shown in figure 15 . The value of $A$ obtained in this way was $4.1 \%$ with $E$ in $\mathrm{GeV}$.

The analysis was repeated including the response of all cells. This result was analysed in two ways. In the first case, $A$ was set to the single cell result of $4.1 \%$ and $B$ to $50 \mathrm{MeV}$. Then the constant term $C$, the only free parameter in the fit, was determined to be $0.4 \%$. The result of the fit is shown in figure 16 as the energy dependence of the energy resolution. In the second case, $B$ was set to $50 \mathrm{MeV}$ and both the statistical and the constant term were left free resulting in a statistical term of $A=4.7 \%$ and a constant term of $C=0.2 \%$. All results are summarized in table 3 .

\begin{tabular}{|l|c|c|c|}
\hline & $A(\%)$ & $B(\mathrm{MeV})$ & $C(\%)$ \\
\hline central cell only & 4.1 & set to 50 & set to 0.0 \\
all cells & set to 4.1 & set to 50 & 0.4 \\
all cells & 4.7 & set to 50 & 0.2 \\
\hline
\end{tabular}

Table 3: Summary of fit results to the energy resolution after corrections

\subsubsection{Material Thickness in Front of Calorimeter}

To obtain a good energy resolution, the material thickness in front of the active part of the calorimeter seen by the incoming particles has to be kept at a minimum. The 


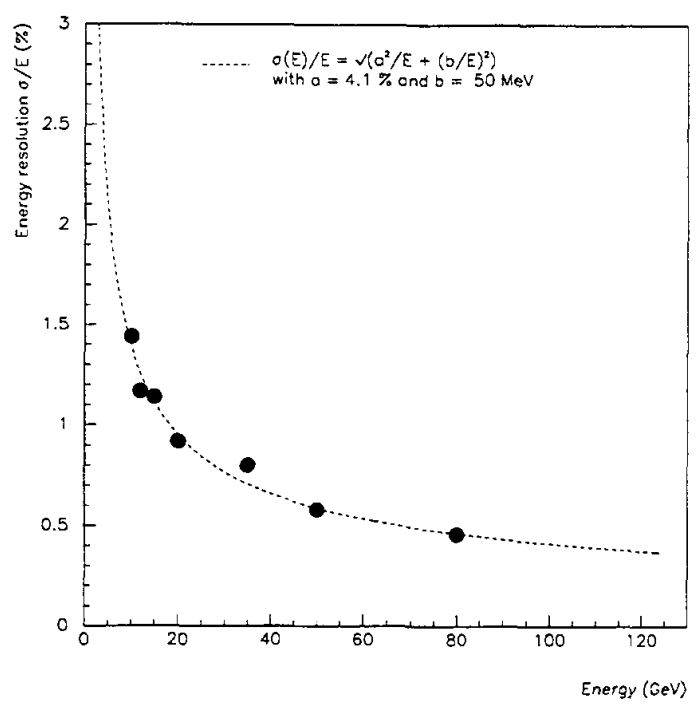

Figure 15: Energy resolution of the prototype calorimeter as measured for a single cell.

total thickness of front material during the test beam data taking was about $0.3 X_{0}$. To study the effect of additional material, data were taken with aluminum plates of different thicknesses $\left(0.5,1.0\right.$ and $\left.1.5 X_{0}\right)$ in front of the calorimeter and for electron energies of 10 and $50 \mathrm{GeV}$. The calorimeter was tilted by $26 \mathrm{mrad}$. Figure 17 shows the energy resolutions which were measured. These resolutions are corrected for cell-to-cell variations of the calorimeter response but not for non-uniformities on a finer scale as for the final energy resolution result. For comparison, five different resolution curves are drawn in the figure $\left((\sigma / E)^{2}=A^{2} / E+0.050^{2} / E^{2}+0.005^{2}\right.$, with $\mathrm{A}=0.03,0.04,0.05,0.06$ and $0.085, \mathrm{E}$ in $\left.\mathrm{GeV}\right)$.

\subsubsection{Temperature Dependence of the Calorimeter Response}

One possible source of systematic uncertainty is a density variation caused by a temperature gradient in the liquid krypton. In order to study the effect of temperature differences, data were taken with the same beam energy at two different temperatures, by adjusting the pressure in the cryostat accordingly. The temperature in the liquid was derived from the pressure.

Table 4 shows the temperature dependence of the calorimeter response at three different energies. As expected the current increases when the temperature is lowered. The systematic error given for the current values was determined by comparing different runs with identical running conditions and does not include the temperature uncertainty. The error quoted on the current change/temperature change ratio is the total error including all systematic errors.

\subsubsection{High Voltage Dependence of the Calorimeter Response}

While most of the data were taken with the high voltage set at $3 \mathrm{kV}$, some runs were taken at high voltages of $3.5,4.0,4.5$ and $5 \mathrm{kV}$. The initial current measured with the calorimeter increased linearly with the voltage, to better than $0.5 \%$ accuracy, following 


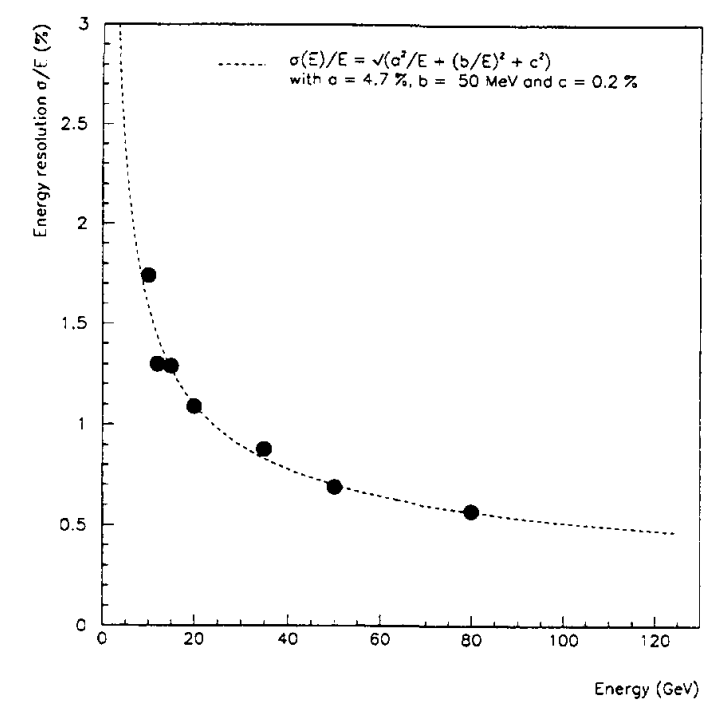

Figure 16: Energy resolution of the prototype calorimeter as measured for different electron energies.

\begin{tabular}{|c|c|c|c|}
\hline $\begin{array}{c}\left(I_{\text {cold }}-I_{\text {nom }}\right) / I_{\text {nom }} \\
(\%)\end{array}$ & $\begin{array}{c}\Delta T \\
\left({ }^{\circ} \mathrm{K}\right)\end{array}$ & $\begin{array}{c}(\Delta I / I) / \Delta T \\
\left(\% /{ }^{\circ} \mathrm{K}\right)\end{array}$ & $\begin{array}{c}\text { Energy } \\
(\mathrm{GeV})\end{array}$ \\
\hline $3.22 \pm 0.17 \pm 0.2$ & $3.2 \pm 0.2$ & $1.01 \pm 0.10$ & 12 \\
$2.31 \pm 0.01 \pm 0.2$ & $2.8 \pm 0.2$ & $0.83 \pm 0.09$ & 35 \\
$2.43 \pm 0.01 \pm 0.2$ & $2.9 \pm 0.2$ & $0.84 \pm 0.09$ & 50 \\
\hline
\end{tabular}

Table 4: Relative current increase for a given temperature decrease of the liquid krypton. $I_{\text {nom }}$ denotes the current measured at the nominal temperature setting, $I_{\text {cold }}$ the current measured at lower temperature.

the relation $\Delta I / I=0.22 \Delta V / V$. The energy resolution showed no significant high voltage dependence.

\subsection{Space Resolution}

For the reconstruction of $K^{0} \rightarrow \pi^{0} \pi^{0}$ decays with subsequent $\pi^{0} \rightarrow \gamma \gamma$ decays, a space resolution of $1 \mathrm{~mm}$ or better is necessary. Furthermore, good photon separation is required to reduce background from the accidental overlap of showers.

The space resolution of the prototype calorimeter has been obtained by comparing the position of the centre of gravity (COG) of the shower in the calorimeter with the electron impact point as reconstructed from the hits in the drift chambers. The COG has been calculated using the peak sensing $\mathrm{ADC}$ information in a shower box of $3 \times 3$ calorimeter cells. Before determining the space resolution, the COG has been corrected for its nonlinear dependence on the impact point inside a cell as reconstructed with the drift chambers.

Figure 18 shows the energy dependence of the space resolution in the $\mathrm{X}$ - and $\mathrm{Y}$ view as measured for the calorimeter orientation of $0 \mathrm{mrad}$ and $26 \mathrm{mrad}$. As expected, the resolution in the $\mathrm{Y}$-view is nearly independent of the orientation whereas the resolution 


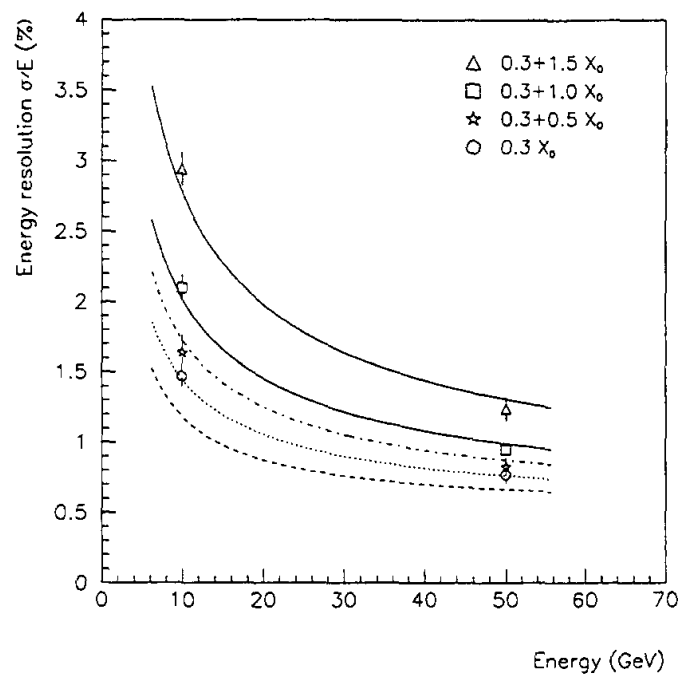

Figure 17: Energy resolution of the prototype calorimeter for different thicknesses of material in front of the calorimeter. The data were taken with the $26 \mathrm{mrad}$ orientation of the calorimeter.

in the $\mathrm{X}$-view is significantly worse at $26 \mathrm{mrad}$ than at $0 \mathrm{mrad}$. The improved uniformity of the calorimeter response as discussed in section 4.1.1 outweighs the deterioration of the space resolution which is expected to be less pronounced for the planned zig-zag structure.

The space resolution can be described by

$$
\sigma=\frac{A}{\sqrt{E[\mathrm{GeV}]}} \oplus B .
$$

Table 5 shows the fit results for $\mathrm{A}$ and $\mathrm{B}$.

\begin{tabular}{|c|c|c|c|}
\hline Orientation & View & $\mathrm{A}(\mathrm{mm})$ & $\mathrm{B}(\mathrm{mm})$ \\
\hline $0 \mathrm{mrad}$ & $\mathrm{X}$ & 4.5 & 0.3 \\
& $\mathrm{Y}$ & 4.8 & 0.6 \\
\hline $26 \mathrm{mrad}$ & $\mathrm{X}$ & 4.3 & 1.1 \\
& $\mathrm{Y}$ & 4.7 & 0.7 \\
\hline
\end{tabular}

Table 5: Space resolution results for the prototype calorimeter.

\subsubsection{Beam Intensity Dependence of the Energy and Space Resolution}

Most of the data were collected with beam intensities in the range of 100 to 10000 particles $/ 60 \mathrm{~cm}^{2}$ per $2.5 \mathrm{~s}$ SPS burst and beam energies between 10 and $80 \mathrm{GeV}$. For 

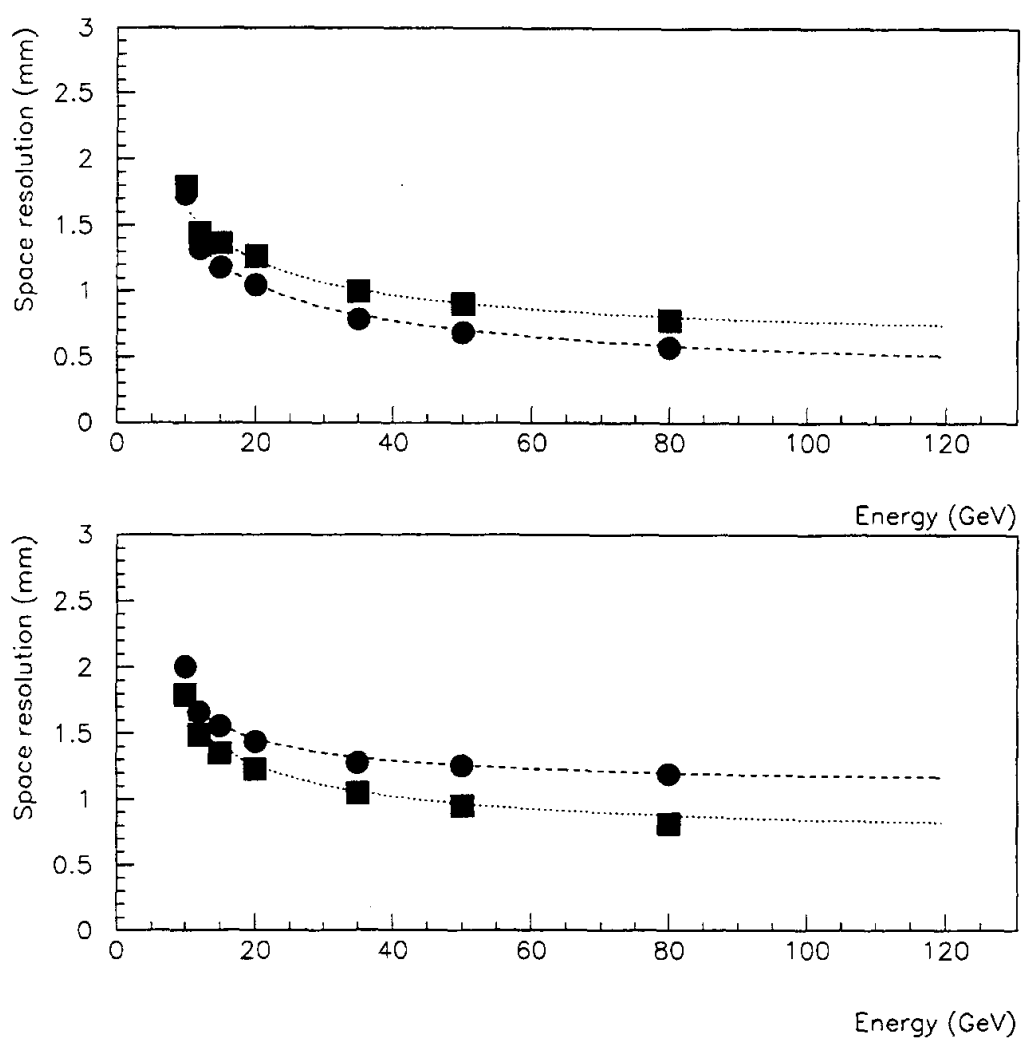

Figure 18: Space resolution of the prototype calorimeter. The upper graph shows the result for $0 \mathrm{mrad}$ orientation, the lower for $26 \mathrm{mrad}$ orientation. Full circles represent the resolution in the $\mathrm{X}$-view, full square boxes in the $\mathrm{Y}$-view.

these data, no significant dependence of the energy or space resolution on the beam intensity has been measured. These intensities cover the running conditions expected for the planned experiment.

At higher intensity, for values equivalent to up to 60000 particles of $50 \mathrm{GeV}$ per burst (data taken with the prototype calorimeter tilted by $13 \mathrm{mrad}$ with respect to the beam axis), a degradation of the energy resolution was observed. This is predominantly due to space charge effects leading to a dependence of the output signal on the shower position between electrodes. At the highest intensity, the yield at the anode was $6 \%$ smaller than the yield at the cathode, to be compared to a typical difference of less than $2 \%$ measured at normal intensity. Furthermore, the effect depended on the time within the spill. At the beginning of the burst the uncorrected energy resolution was similar to the value measured at normal intensity. It worsened for the first $\approx 0.6 \mathrm{~s}$, plateauing at a full width at half maximum value approximately twice as large as the initial one for the rest of the burst. It appears that space-charge in the liquid krypton contributes significantly to these effects, and we estimate a value of $\approx 0.5 \mathrm{~cm}^{2} \mathrm{~s}^{-1} \mathrm{kV}^{-1}$ for the mobility of positive ions in the liquid krypton. These effects only appear at intensities well beyond those expected for the final running conditions. 


\subsection{Time Resolution}

Time resolution of better than $1 \mathrm{~ns}$ is required to detect $K_{L}$ and $K_{S}$ decays concurrently and distinguish the $K_{L}$ and $K_{S}$ by the coincidence with a signal from a tagging counter system in the proton beam line leading to the $K_{S}$ target [1].

A readout cell electrically forms a transmission line which is approximately terminated by the input impedance of the preamplifier in the frequency range of interest. The signal propagates in the direction of shower development with a velocity of $4 \mathrm{~ns} / \mathrm{m}$. The timing of the signal is affected by longitudinal shower fluctuations and pulse shape variations caused by the lateral shower distribution. If the time of the signal is derived from the leading edge, contributions to the time resolution due to shower fluctuations and electronic noise [13] are expected to be well below 300 ps for energies above $10 \mathrm{GeV}$. Other contributions may originate from the limited pulse height resolution and phase stability of the FADC system employed.

The time resolution of the protoype was determined from data taken with the 40 $\mathrm{MHz}$ linear 8 bit FADC system ${ }^{7)}$. Only the inner 80 cells were equipped with FADCs. To increase the dynamic range, up to three FADCs with different gain settings $(\times 10, \times 3.3$, $\times 1.1$ ) were connected to the same cell. Out of the 80 cells with FADCs, 16 were equipped with two FADCs and 16 with three FADCs in parallel. For each event and channel, 48 FADC samples covering $1.2 \mu$ s were written to tape. The relative time between the clock of the FADC system and the event trigger was measured with a 50 ps TDC ${ }^{8}$ ). The energy and space resolutions measured with the FADC system gave comparable results to the conventional ADC system when restricted to 80 cells [14].

The time of a pulse is defined as the time at which the leading edge of the FADC output pulse reached half its maximum height. The best time resolution was obtained by calculating the weighted mean of the times measured in the cells in a $3 \times 3$ box around the cell with the maximum energy deposit. The weight for each cell was given by the square of the amplitude measured in the cell.

Figure 19 shows the energy dependence of the time resolution. After cell-to-cell and pulse height corrections, the resolution is better than $0.6 \mathrm{~ns}$ for all energies above $10 \mathrm{GeV}$. Energy independent contributions to the time resolution come from the time resolution of the trigger (180 ps, see also section 3.2), the accuracy of the FADC clock phase measurement (100 ps) and non-uniformities in the calorimeter response. The weak dependence of the time resolution on energy indicates that the limit given by shower fluctuations, electronic noise and pulse height resolution has not yet been reached. The exact shape of the energy dependence shown in figure 19 is due to the different resolutions of the FADC types since at each energy different FADCs with different gain settings contribute to the measurement. There are no significant non-Gaussian tails seen in the time distributions. As an example, the time distribution for $35 \mathrm{GeV}$ is shown in figure 20 .

\footnotetext{
7) Dr. Bernd Struck, STR750 Flash-ADC System.

8) LeCroy Research Systems, Model 2228A
} 


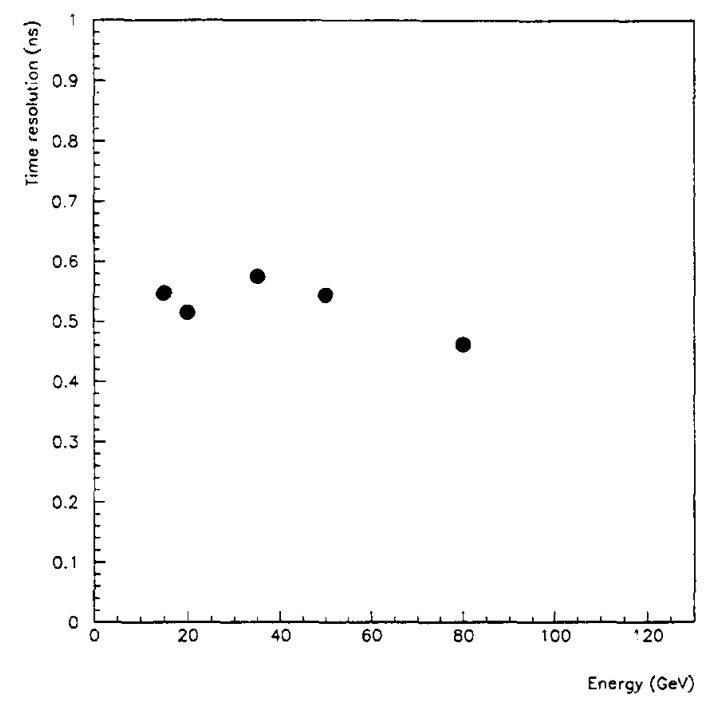

Figure 19: Time resolution of the prototype calorimeter.

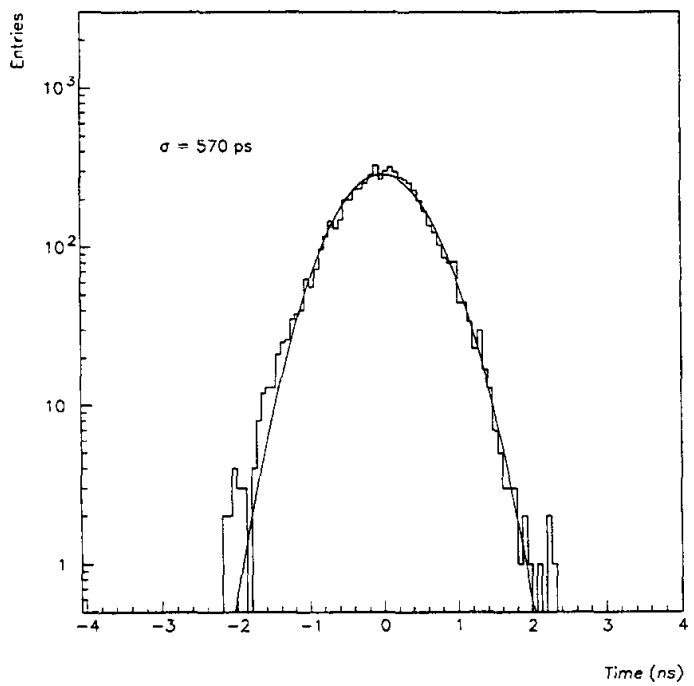

Figure 20: Time distribution of the prototype calorimeter at $35 \mathrm{GeV}$. 


\subsection{Response to Muons}

Data with incident muons with a nominal momentum of $50 \mathrm{GeV} / c$ have been collected to study the calorimeter response to minimum ionizing particles and to check the current to energy factor of the calorimeter. The energy loss of the muon was obtained by adding signals in a shower box of $3 \times 3$ cells around the cell hit. To select muons with the track length contained in a single cell, the contribution of the cell hit was required to be at least $80 \%$ of the reconstructed energy. The spectrum of the reconstructed energy is shown in figure 21 . The current scale $(\mu \mathrm{A})$ is determined by means of the electronic calibration. The electronic noise contribution is negligible due to the small number of channels summed together.

The most probable value of the energy loss for a minimum ionizing particle (mip) is obtained by means of a fit of two Gaussians, one for each side of the peak, yielding a value

$$
1 \mathrm{mip}=(1.147 \pm 0.001) \mu \mathrm{A} .
$$

A Monte Carlo simulation [15] of all the processes involved in the energy loss of $50 \mathrm{GeV}$ muons in the calorimeter gave the most probable value of energy loss as ( $475 \pm$ 10) $\mathrm{MeV}$. From these two numbers the current to energy conversion for muons has been computed :

$$
\frac{I[\mu \mathrm{A}]}{E[\mathrm{GeV}]}=\frac{1.147 \mu \mathrm{A}}{0.475 \mathrm{GeV}}=(2.41 \pm 0.05) \frac{\mu \mathrm{A}}{\mathrm{GeV}} .
$$

The value given by the electron beam calibration $(2.1 \mu \mathrm{A} / \mathrm{GeV})$ is about $15 \%$ smaller. The difference comes from shower box leakage and losses in passive materials, both of which are larger for an electron shower.

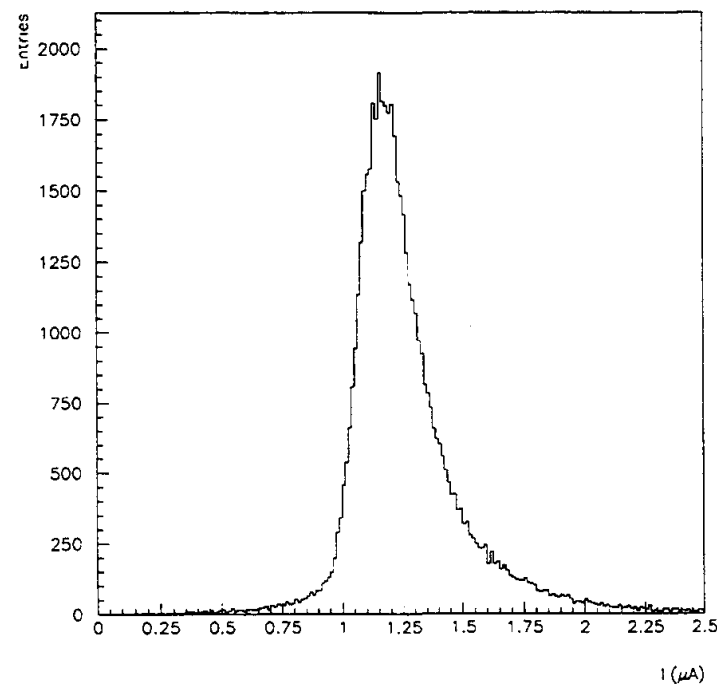

Figure 21: Energy loss spectrum for muons in the NA48 prototype calorimeter.

\section{Conclusion}

A prototype electromagnetic calorimeter containing about 1801 liquid krypton has been built and tested. For electron energies larger than $20 \mathrm{GeV}$, we have measured an 
energy resolution better than $1 \%$, a space resolution better than $1 \mathrm{~mm}$, and a time resolution better than $600 \mathrm{ps}$. The electronic noise, summed over the cluster of cells used in the energy measurement, was $46 \mathrm{MeV}$.

\section{Acknowledgements}

We gratefully acknowledge the effort of J. Armand, T. DeOliveira, G. Dubail, J. Fersurella, Y. Grandjean, P. LeCossec, K. Ley, P. Schilly, W. Schüller, R. Truhan, B. Überschär, P. Wicht and W. Wilkens in the construction and installation of the prototype calorimeter and the readout electronics, and the contribution of F. Louis, G. Tarte, E. Pasquetto and P. Roth for setting up the chambers. We also appreciate the technical support of G. Pagani and L. Zaccarelli from INFN Pisa. We thank L. Bertanza, A. Bigi, M. Calvetti, R. Carosi, R. Casali, M. DeBeer, R. DeWolf, J.-P. Huber, D. Munday, A. Nappi, A. Parker, F. Petrucci, G. Pierazzini and T. White for their help during setup and data taking. We thank the technical support groups in AT, ECP, PPE and SL divisions at CERN for their help. We acknowledge fruitful discussions with V.Radeka on the design of the analogue circuitry.

After the completion of this paper, we became aware of the excellent work of O. Benary et al. $[16,17]$ who have constructed and tested an accordion electromagnetic calorimeter with liquid krypton and argon.

\section{References}

[1] G.D.Barr et al., CERN/SPSC/90-22/P253.

[2] H.Burkhardt et al., NIM A 268 (1988) 116.

H.Burkhardt et al., PL B206 (1988) 169.

G.D.Barr et al., PL B317 (1993) 233.

[3] C.Cerri et al., NIM 227 (1984) 227.

[4] V.Radeka et al., NIM A 265 (1988) 228.

[5] T.Stemmler, diploma thesis, Darmstadt, 1993, to be published.

[6] D.Schiuma, diploma thesis, Munich, 1993.

[7] P.Buchholz, NIM A 316 (1992) 1.

[8] G.D.Barr et al., NIM A 323 (1992) 393.

[9] A.Bettini et al., NIM A 305 (1991) 177.

[10] B.Aubert et al., CERN/DRDC/90-31 DRDC/P5.

[11] B.Hallgren, G.Laverrière, CERN/ECP 91-33.

[12] B.Gorini, diploma thesis, Pisa, 1993.

[13] H.Wahl, NIM 25 (1964) 247.

[14] G.Viehhauser, diploma thesis, Technical University, Vienna, 1993.

[15] R.Brun et al., CERNLIB GEANT Monte Carlo System.

[16] O.Benary et al., NIM A 332 (1993) 78.

[17] O.Benary et al., BNL 49526 (1993). 
